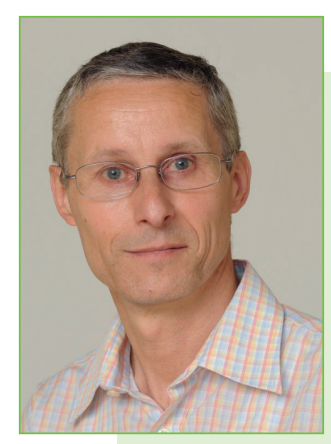

\title{
Länger gesund leben!
}

Mit dieser Überschrift, liebe Leserinnen und Leser, möchte ich Sie auf eine Droge aufmerksam machen, die trotz jahrtausendlanger volksheilkundlicher Anwendung erst in den letzten Jahrzehnten auch im Westen zunehmende Beachtung und Anerkennung findet. Die unfermentierten Blätter von Camellia sinensis (L.) Kuntze sind unter der Bezeichnung Grüntee oder grüner Tee im Tee- oder Lebensmittelgeschäft erhältlich - Extrakte auch in der Apotheke. Insgesamt konsumierten die deutschen Verbraucher davon im vergangenen Jahr rund 4500 Tonnen Tendenz steigend. Wie im Fernen Osten, so nutzen auch die Menschen bei uns dieses Getränk als Genussmittel und gleichzeitig als Phytotherapeutikum zur Gesunderhaltung. Dazu einige Fakten aus den letzten Jahren:

Eine Langzeitstudie japanischer Forscher zeigt, dass Teetrinker, die regelmäßig grünen Tee konsumierten, weit weniger oft an gefährlichen HerzKreislauf-Erkrankungen starben als Nicht-Teetrinker. Elf Jahre lang dokumentierten die Ärzte Todesfälle und deren Ursachen in Zusammenhang mit diesem Getränk an mehr als 40000 Japanern im Alter zwischen 40 und 79 Jahren (JAMA 2006; 296: 1255-1265). Das Fazit der Untersuchungen: Teetrinker hatten eine um 16\% niedrigere Sterberate als diejenigen, die keinen oder kaum Tee tranken; allerdings sollte man täglich mindestens 5 Tassen davon trinken und auch generell gesund leben. Bestätigt wurden diese Ergebnisse noch einmal in einer weiteren Studie aus Japan, in der bei 83000 Japanern über ca. 13 Jahre hinweg die Trinkgewohnheiten bezüglich grünem Tee untersucht wurden. Auch hier zeigte sich, dass die Gefahr, einen Schlaganfall zu erleiden, negativ mit der Menge konsumierten Grüntees korreliert: Bei Studienteilnehmern, die täglich 2-3 Tassen grünen Tee tranken, war das Risiko um 14\% niedriger, bei mindestens 4 Tassen verringerte es sich um 20\% (Stroke 2013; 44: 1369-1374). Wenn gleichzeitig das umfassendste Werk über den Gesundheitszustand und das Gesundheitsverhalten der 18- bis 79-Jährigen, die "Studie zur Gesundheit Erwachsener in Deutschland “ (DEGS) des Robert Koch-Instituts, zeigt, dass Herz-Kreislauf-Erkrankungen an vorderer Stelle in der Mortalitätsstatistik stehen, dann machen Grüntee-Trinker alles richtig, um möglichst lange gesund zu bleiben.
Das aromatische Getränk beinhaltet eine Vielzahl von pharmakologisch aktiven Substanzen, die neben dem Schutz der Blutgefäße auch insgesamt den Organismus vor schädlichen Einflüssen schützen. So besitzt Grüntee antibakterielle, antifungale und antivirale Eigenschaften, die die Gesundheit des Magen-Darm-Traktes erhalten. Auch bei metabolischem Syndrom ist das Getränk an der Normalisierung der Blutlipid- und Glukosewerte beteiligt. Eine Reihe von Untersuchungen hat gezeigt, dass das Trinken von grünem Tee die Entstehung von neurodegenerativen Erkrankungen bremsen kann. Damit dürfte dieses Getränk in die Liga der "Allheilmittel» aufsteigen - wissenschaftlich begründet und preiswert für alle Menschen verfügbar.

Bei aller Skepsis eines kritischen Lesers muss man dem Grüntee konzedieren, dass es bezüglich seiner pharmakologischen und protektiven Eigenschaften heute keine grundsätzlichen Diskussionen mehr gibt. Das weltweit am häufigsten aufgenommene Getränk vereint die Eigenschaften eines optimalen Durstlöschers mit denen eines sinnvoll zusammengesetzten Naturstoff-Cocktails, der mild aber effektiv in die menschliche Physiologie eingreift und bei häufigem Gebrauch immer besser wirkt. Welche $\mathrm{Zu}$ bereitung kann dies schon von sich behaupten! Natürlich gehört auch eine gesunde Lebensweise dazu, aber diese Einsicht teilen zunehmend mehr Menschen - nicht nur Teetrinker.

Wenn Sie die nächste Tasse Grüntee trinken, dann vielleicht in dem Bewusstsein, etwas für die eigene gesunde Langlebigkeit zu tun. Sie können den Tee aber auch nur genießen und daran denken, was bereits der sagenumwobene chinesische Kaiser Shen Nung meinte: "Tee weckt den guten Geist und weise Gedanken. Er erfrischt das Gemüt. Bist du niedergeschlagen, so wird Tee dich ermuntern." Ein Ratschlag, den ich mir täglich mehrfach zu Herzen nehme!

Matthias F. Melzig 